\title{
Designing Sparse Wireless Multi-hop Networks
}

\author{
Srinath Perur \\ Advisor: Sridhar Iyer \\ KReSIT, IIT Bombay \\ Email: [srinath, sri]@it.iitb.ac.in
}

\section{INTRODUCTION}

Wireless Multi-hop Networks (WMNs) are decentralized, infrastructure-less networks enabled by cooperative multi-hop routing among the participating nodes. Packet radio networks, mobile ad hoc networks, and sensor networks are instances of WMNs. A sparse WMN is one in which connectivity with high probability is not ensured. Such a network can arise in various ways: a vehicular ad hoc network in an area with low traffic density, an initially connected sensor network after some of its nodes have failed, and an ad hoc communications network that is being deployed incrementally can all be sparse networks. In constrained deployment scenarios, we may even wish to deploy a multi-hop network that trades off connectivity for deployment cost. There is work [1] that shows that tolerating some sparseness (requiring only $90 \%$ of nodes to be in the same connected component) results in a significant reduction in the required transmission range of nodes.

The ability to evaluate tradeoffs between deployment parameters is important in WMNs. Gupta and Kumar showed in [2] how throughput per source-destination pair in a WMN decreases as node density increases. Grossglauser and Tse in [3] later showed that mobility could be exploited to achieve a tradeoff between throughput and delay. This would allow throughput to be maintained almost constant even with increasing node density. Similarly, a tradeoff has also been achieved between connectivity and delay. Delay tolerant routing [4] and Message Ferrying [5] are representative of work that uses node mobility to achieve asynchronous communication between disconnected nodes in sparse networks. Connectivity is also a limiting factor in sparse WMNs: in the absence of paths between nodes, issues of interference and network capacity become irrelevant. This motivates the study of appropriate metrics and tools for the design of sparse WMNs.

\section{ReACHABILITy}

The extent of communication possible in a WMN is usually described by the connectivity of the network. Connectivity is defined as the probability that all nodes in the network form a single connected component. Much work in the area of WMNs has revolved around connectivity, and how to ensure it through appropriate choices of deployment parameters such as number of nodes and transmission range of nodes. However, connectivity is unsuitable when applied to sparse networks since i) it is not indicative of the actual extent of communication possible in the WMN; and ii) it is unresponsive to fine changes in network parameters. We propose the fraction of connected node pairs as a more appropriate measure of the communication capabilities of a sparse network, and call this term reachability. Figure 1 is obtained from simulations, and plots the growth of Reachability and Connectivity as the transmission range, $R$, increases for 60 static nodes distributed uniformly at random in a $2000 \mathrm{~m} \times 2000 \mathrm{~m}$ area. In this case, when reachability is 0.4 , meaning $40 \%$ of node pairs are connected, connectivity is still at zero. Further, using only connectivity here is clearly inappropriate since the connectivity curve would lead us to believe that increasing $R$ from 50 to any value less than 320 would have no effect on the extent of communication supported by the network.

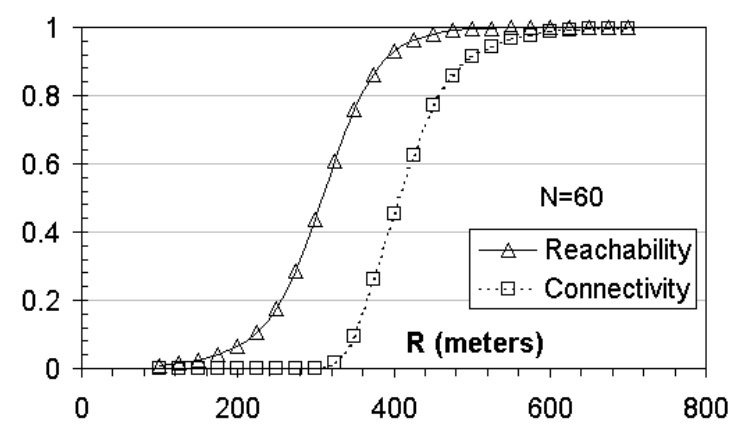

Fig. 1. Increasing R, no mobility

The reachability of a static network is defined as the fraction of connected node pairs in the network. It is a property of the network graph, with no assumptions made regarding the distribution of nodes. Using this definition we can calculate reachability for a network of $N$ nodes as:

$$
\text { Reachability }=\frac{\text { No. of connected node pairs }}{\left(\begin{array}{c}
N \\
2
\end{array}\right)}
$$

\section{SIMRAN}

Simran ${ }^{1}$ is a simulator we have developed for studying topological properties of WMNs. Simran takes as input a scenario file with initial positions and movement scripts of nodes, and generates a trace file containing metrics of interest such as average number of neighbors, averaged shortest path lengths over all pairs of nodes, reachability, connectivity, and number and size of connected components. Simran is also supported by a number of smaller programs for generating scenario files, managing large simulations, and for analyzing results. Simran also supports topological simulation of networks with asynchronous communication, and can be used to evaluate design tradeoffs in sparse WMNs. Figures 1 and 2

\footnotetext{
${ }^{1}$ Available from http://www.it.iitb.ac.in/ srinath/simran/
} 
are generated from results of simulations in Simran. Note that in Fig. 2, almost $80 \%$ of node pairs have a path connecting them before connectivity rises above zero.

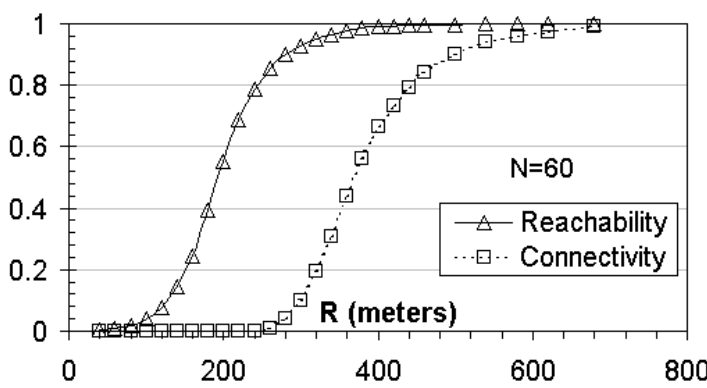

Fig. 2. Increasing R, with mobility and asynchronous communication

\section{Characterizing ReACHABILITy}

Our network model is as follows: $N$ nodes, each with a transmission range of $R$ are distributed uniformly at random in a square area of side $l ; r=R / l$ is the normalized transmission range, and $M$ denotes the mobility parameters. We denote the value of reachability for such a network as $R c h_{N, r}^{M}$. In the static case, we represent it as $R c h_{N, r}$. If the $N$ nodes form $k$ components with $m_{i}$ nodes in the $i^{t h}$ component, we can rewrite Eqn. 1 as

$$
\operatorname{Rch}_{N, r}=\frac{\sum_{i=1}^{k}\left(\begin{array}{c}
m_{i} \\
2
\end{array}\right)}{\left(\begin{array}{c}
N \\
2
\end{array}\right)}=\frac{\sum_{i=1}^{k} m_{i}\left(m_{i}-1\right)}{N(N-1)}
$$

It may be possible to obtain asymptotic bounds for $R c h_{N, r}$, but since sparse networks often involve small Numbers of nodes, we are particularly interested in characterizations in the finite domain, and chose to model reachability through empirical regression.

We explored data from comprehensive simulations, and found that $R c h_{N, r}$ obeys logistic growth as given by:

$$
\operatorname{Rch}_{N, r}=\frac{1}{1+e^{\alpha_{N}-\beta_{N} r}}
$$

We then:

- conducted extensive simulations to obtain data that represented the growth of $R c h_{N, r}$ from 0 to 1 as $r$ increased, while keeping $N$ fixed;

- used Eqn. 3 as a regression function for simulated data, and obtained the coefficients $\alpha$ and $\beta$ for the corresponding value of $N$-this allowed us to characterize reachability as a function of $r$ for one value of $N$;

- We repeated the above two steps for values of $N$ ranging from 2 to 500 , and performed a second level of regression on the estimated values of $\alpha_{N}$ and $\beta_{N}$.

This gave us a set of equations that allows us to obtain reachability as a function of $N$ and $r$ for values of $N$ ranging from 2 to 500 .

On validating the model, we found that the average relative error in the predicted $R c h_{N, r}$ was $3.5 \%$. We did not observe a single instance when the model was in error by more than 0.05 . We have also extended this model to make it usable up to $N=1000$, but with a larger margin of error. A detailed account of the characterization can be found in [6].

\section{SPANNER}

Spanner ${ }^{2}$ is a design tool that uses the reachability model described in Sec. IV. Given three values from deployment area, $N, R$, and reachability, it computes the fourth.

The assumptions made by Spanner regarding the network model are quite idealized. However, it should be possible to use it for sparse WMN deployment after choosing parameters conservatively. Figure 3 is from data generated by Spanner, and shows the $R$ and $N$ values required to maintain a desired value of reachability.

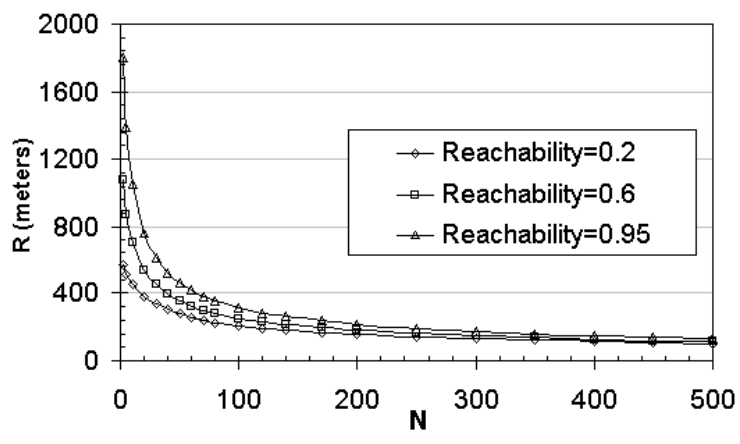

Fig. 3. R vs. $\mathrm{N}$

\section{CONCLUSIONS}

Sparse WMNs can support a significant extent of communication. This is specially true in the presence of mobility coupled with asynchronous communication, as seen in Fig. 2. However, existing tools may not be sufficient for exploring the design of such networks. We presented a metric, reachability, its empirical characterization, and two tools for aiding the design of sparse WMNs. A detailed account of their use in facilitating tradeoffs for sparse WMN deployment can be found in [7].

\section{REFERENCES}

[1] P. Santi and D. M. Blough, "The critical transmitting range for connectivity in sparse wireless ad hoc networks," IEEE Transactions on Mobile Computing, vol. 2, no. 1, pp. 25-39, 2003.

[2] P. Gupta and P. R. Kumar, "The capacity of wireless networks," IEEE Transactions on Information Theory, vol. 46, no. 2, pp. 388-404, March 2000.

[3] M. Grossglauser and D. Tse, "Mobility increases the capacity of ad-hoc wireless networks," in IEEE INFOCOM, vol. 3, 2001, pp. 1360-1369.

[4] S. Jain, K. Fall, and R. Patra, "Routing in a delay tolerant network," in ACM SIGCOMM '04, 2004, pp. 145-158.

[5] W. Zhao, M. Ammar, and E. Zegura, "A message ferrying approach for data delivery in sparse mobile ad hoc networks," in ACM MobiHoc '04, 2004, pp. 187-198.

[6] S. Perur and S. Iyer, "Characterization of a connectivity measure for sparse wireless multi-hop networks," To appear in Proceedings of the Workshop on Wireless Ad hoc and Sensor Networks, held in conjuction with ICDCS 2006, Lisboa, Portugal, July 2006.

[7] — "Sparse multi-hop wireless for voice communication in rural India," Proceedings of the 12th National Conference on Communications, NCC 2006, New Delhi, India, pp. 534-538, January 2006.

\footnotetext{
${ }^{2}$ Sparse network planner: http://www.it.iitb.ac.in/ srinath/tool/rch.html
} 